\title{
Interplay between Internal and External Electric Field Studied by Photoluminescence in InGaN/GaN Light Emitting Diodes
}

\author{
G. Staszczak ${ }^{a, *}$, A. Khachapuridze ${ }^{a}$, S. Grzanka ${ }^{a, b}$, R. Czernecki $^{a, b}$, \\ R. Piotrzkowski ${ }^{a}$, P. Perlin ${ }^{a, b}$ And T. Suski ${ }^{a}$ \\ ${ }^{a}$ Institute of High Pressure Physics PAS, Sokołowska 29/37, 01-142 Warszawa, Poland \\ b TopGaN, Sokołowska 29/37, 01-142 Warszawa, Poland
}

\begin{abstract}
We have studied a series of polar InGaN/GaN light emitting diodes, consisting of either a blue (440-450 nm) quantum well, or combination of blue and violet $(410 \mathrm{~nm})$ quantum wells (with indium content $18 \%$ and $10 \%$, respectively). The blue quantum well was always placed close to $p$-type region of the particular LED. We found that the electroluminescence induced by low current is characterized by light emission from the blue quantum well only. In comparison, optical excitation of our LEDs leads to light emission with energies characteristic either for blue and/or violet quantum wells. The corresponding microphotoluminescence spectra evolve depending on external polarization and variable light intensity of excitation supplied by $\mathrm{He}-\mathrm{Cd}$ laser. Interplay between built-in electric field and externally applied polarization/screening decides about the band structure profiles and thus radiative recombination mechanisms.
\end{abstract}

PACS: 78.60.Fi, 78.67.De, 85.60.Bt, 85.60.Jb

\section{Introduction}

Efficient light emitting diodes (LEDs) and laser diodes (LDs) consist usually of $\operatorname{In}_{x} \mathrm{Ga}_{1-x} \mathrm{~N} / \mathrm{GaN}$ multi quantum wells (MQWs) in their active region. However, due to the presence of internal electric fields in polar structures, devices with $\mathrm{InGaN} / \mathrm{GaN}$ QWs give a reduction in the overlap between electron and hole wave functions, leading to a decrease of the emitted light intensity. In nitride LEDs and LDs, an additional electric field originating from the $p-n$ junction also contributes to the band edges profiles of the device structure and light emission from each individual QWs can be tuned by an externally applied voltage.

We focus only on experimental method in this work since there are a large number of factors that influence the energy and light emission efficiency making a full understanding of the InGaN/GaN LEDs and LDs behavior very difficult $[1,2]$. Furthermore, the capability of computer simulations is limited since the magnitude of large number of parameters describing nominally the studied nitride structures is not well known.

\section{Experimental procedure}

To improve the understanding of LEDs behavior we have studied a series of polar InGaN/GaN light emitting diodes grown by metalorganic vapor phase epitaxy

\footnotetext{
* corresponding author; e-mail: staszczak@unipress.waw.pl
}

(MOVPE) on sapphire substrate. The LEDs studied consist of an active region consisting of either blue $(\lambda \approx$ 440-450 nm) QW, or a combination of blue and violet $(\lambda \approx 410 \mathrm{~nm})$ QWs (with indium content $18 \%$ and $10 \%$, respectively). The blue QW was always placed close to $p$-type region of the particular LED. In this work, we examined LEDs with (i) 1 blue QW ("1" LED, Fig. 1a), (ii) 1 blue and 1 violet QWs (" $1+1$ " LED), and (iii) 1 blue and 2 violet QWs (" $2+1$ " LED, Fig. 1b). Since we are aware of the strong influence of an AlGaN electron blocking layer (EBL) on the performance of InGaN/GaN LEDs, we decided to use a GaN EBL, a simpler structure to understand [2].

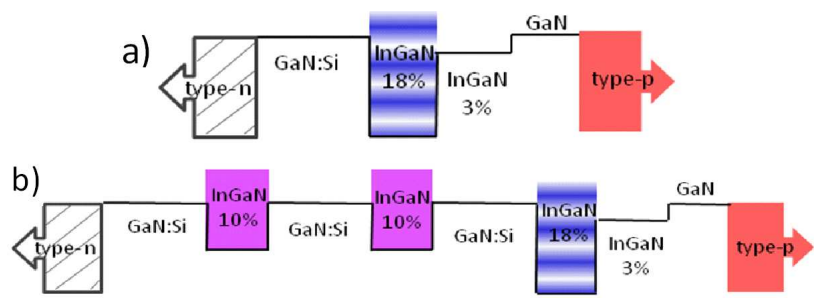

Fig. 1. Conduction band scheme of the investigated LED structures: (a) with 1 blue QW $(\lambda=440-450 \mathrm{~nm})$, (b) with 1 blue and 2 violet QWs $(\lambda=410 \mathrm{~nm})$.

To study light emission from the corresponding LEDs $n$-type and $p$-type ohmic contacts were deposited on the sample. The $p$-type contact had a small rectangular opening to allow access for microphotolumi- 


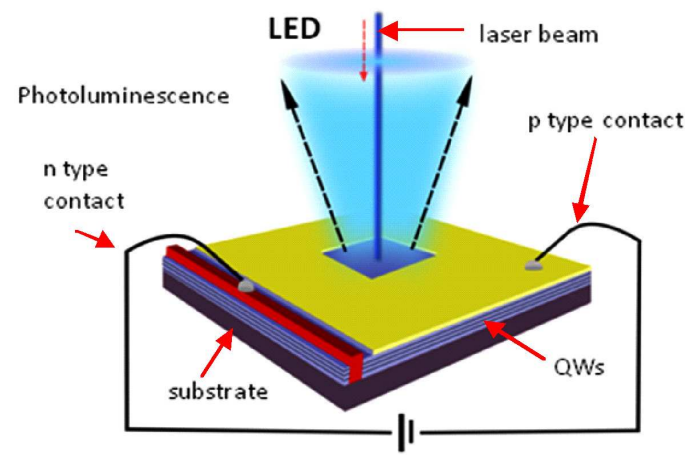

Fig. 2. Schematic illustration of the $\mu \mathrm{PL}$ measurements. Electrical contacts to $p$-type and $n$-type are also shown.

nescence $(\mu \mathrm{PL})$ studies or emitted light from electroluminescence (EL). A schematic illustration of the $\mu \mathrm{PL}$ measurements is given in Fig. 2. The EL was measured at a bias voltage of $+3.2 \mathrm{eV}$ (with a supplied current $20 \mathrm{~mA}$ ); whereas the $\mu \mathrm{PL}$ was studied for a range of biases between $-7 \mathrm{~V}$ and $+3.2 \mathrm{~V}$. A He-Cd laser $(325 \mathrm{~nm})$ was used to excite the $\mu \mathrm{PL}$. All reported measurements were performed at a temperature of $300 \mathrm{~K}$.

\section{Results and discussion}

We found that in agreement with expectations, the electroluminescence induced by $20 \mathrm{~mA}$ current characterizes light emission from the blue QW that is situated close to the $\mathrm{Mg}$-doped part of the structure. The measured electroluminescence at 440-450 $\mathrm{nm}$ originates from the blue QW that is located close to the $p$-type region. This effect is observed in all LEDs studied here. Figure 3 illustrates this behavior as observed for the case of the " $2+1$ " LED. We believe that this is because holes are injected into the active region and recombine predominantly in the QW close to the $p$-GaN layer [3-5]. We found that electroluminescence intensity produced by "1" LED, " $1+1$ " LED, and " $2+1$ " LED gives a ratio of intensities corresponding roughly to $1: 7: 2$, respectively. It shows that the InGaN/GaN structure corresponding to " $1+1$ " LED represents the most suitable design for optimized electroluminescence performance. We associate this observation with the positive role of the violet QW acting as an "electron cooler", decreasing the kinetic energy of electrons travelling from the $n$ - to $p$-type region of the device. Hence, a relatively large number of the "cool electrons" can be trapped and radiatively recombine in the deeper (blue) QW.

In comparison, photoluminescence shows that the dominant emission is from the QW most distant from $p$-type region. Moreover, a large negative bias voltage eliminates photoluminescence entirely (see Fig. 4).

The combination of electric fields originating from both the $p-n$ junction and the built-in field (caused by spontaneous and piezoelectric polarization contributions) plays

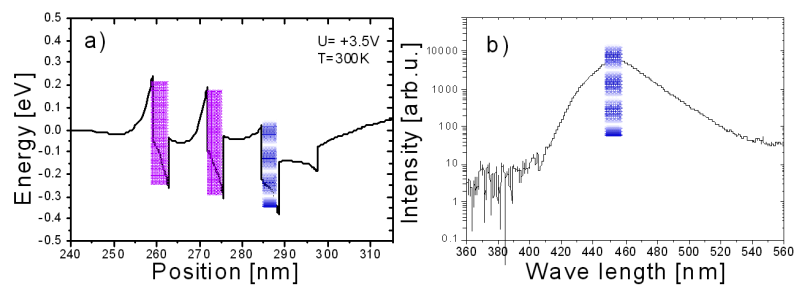

Fig. 3. Conduction band structure of (a) " $2+1$ " LED and (b) EL of " $2+1$ " LED with emission originating from blue QW - situated close to $p$-type region.

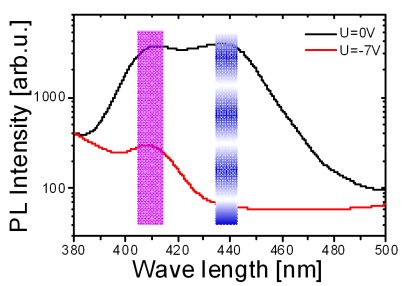

Fig. 4. $\mu \mathrm{PL}$ of " $1+1$ " LED - emitted light originates from blue $(\lambda=440 \mathrm{~nm})$ and violet $(\lambda=410 \mathrm{~nm}) \mathrm{QWs}$ at $0 \mathrm{~V}$. For large negative bias voltages, the light emission from the blue QW disappears.

a crucial role in light emission from QWs of the studied LED structures. Hence, we can tune the effective electric field by applying a bias voltage and/or varying the laser excitation power, i.e., varying the injected carrier densities.

We have performed simple simulations of the photoluminescence dependence on applied bias voltage and intensity of optical excitation in a $\operatorname{In}_{x} \mathrm{Ga}_{1-x} \mathrm{~N} / \mathrm{GaN}$ LED (consisting of one quantum well with In-content of $x \approx$ 0.18 ) using the SIMWINDOWS program. The software solves 2-dimensional drift-diffusion of carriers in which one can also introduce optical generation of carriers. Calculations have been carried out for a simplified $p-i-n$ InGaN/GaN LED structure, where " $i$ " denotes an intrinsic region of LED. We chose the input parameters to be similar to "1" LED with a single $4 \mathrm{~nm} \mathrm{In}_{x} \mathrm{Ga}_{1-x} \mathrm{~N} / \mathrm{GaN}$ QW with $x=0.15$, located at the border between the $n$ - and $i$-regions (and within a distance of $20 \mathrm{~nm}$ from $p$-type region) and the concentration of $\mathrm{Mg}$-acceptors in the $p$-type region and $\mathrm{Si}$-donors in $n$-type region were $N_{\mathrm{A}}=5 \times 10^{19} \mathrm{~cm}^{-3}$ and $N_{\mathrm{D}}=5 \times 10^{18} \mathrm{~cm}^{-3}$, respectively.

The magnitude of the polarization charge was calculated using the SiLENSe program. (Interestingly, the calculated magnitude of built-in electric field for the considered structure is about $2 \mathrm{MV} / \mathrm{cm}$.) Other parameters utilized in the simulation are as follows: absorption coefficient, $10^{5} \mathrm{~cm}^{-1}$; radiative recombination constant, $B_{\mathrm{R}}=10^{-11} \mathrm{~cm}^{3} / \mathrm{s}$ and nonradiative recombination time $\tau_{\mathrm{NR}}=10^{-9} \mathrm{~s}$. The calculated dependence of light intensity versus applied bias voltage (with laser excitation as a parameter) shows that the photoluminescence vanishes 


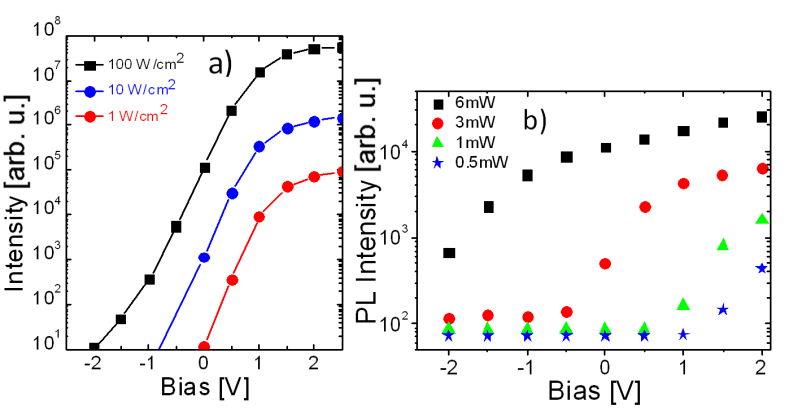

Fig. 5. Evolution of the $\mu \mathrm{PL}$ signal intensity as a function of applied bias voltage with increasing intensity of exciting laser beam for QWs "1". Results of simulations and experiment are shown in Fig. 5a and b, respectively.

when the space charge region is wider (Fig. 5), corresponding to the increase of negative polarization. Summarizing this part of our studies we can see that the experimental results and simulations (Fig. 5b and a, respectively) shows clearly a qualitative agreement.

\section{Summary}

A set of LEDs consisting of QWs emitting light in the blue and violet spectral range was grown by MOVPE method. We found that electroluminescence induced by low currents characterizes light emission from the blue QWs that is located close to the $p$-type region of the LEDs. The corresponding microphotoluminescence spectra evolve depending on external polarization and optical excitation intensity. Interplay between the built-in electric field and the externally applied polarization/screen- ing determines the band structure profile and thus radiative recombination mechanisms. We demonstrate an interesting way of tuning the photoluminescence intensity from single and multi-quantum well LEDs (Figs. 4 and 5). Moreover, we obtained information helpful for optimizing the efficiency of InGaN/GaN LEDs operating at desired spectral range.

\section{Acknowledgments}

This research was supported by the European Union within European Regional Development Fund, through grant Innovative Economy (POIG.01.01.02-00-008/08) and grant of Ministry of Science and Higher Education No. NN202131339.

We thank Dr. Stephen Najda for fruitful discussions and a critical reading of the manuscript.

\section{References}

[1] M. Peter, A. Laubsch, W. Bergbauer, T. Meyer, M. Sabathil, J. Baur, B. Hahn, Phys. Status Solidi A 206, 1125 (2009).

[2] S. Grzanka, G. Franssen, G. Targowski, K. Krowicki, T. Suski, R. Czernecki, P. Perlin, M. Leszczyński, Appl. Phys. Lett. 90, 103507 (2007).

[3] A. David, M.J. Grundmann, J.F. Kaeding, N.F. Gardner, T.G. Mihopoulos, M.R. Krames, Appl. Phys. Lett. 92, 053502 (2008).

[4] J.P. Liu, J.-H. Ryou, R.D. Dupuis, J. Han, G.D. Shen, H.B. Wang, Appl. Phys. Lett. 93, 021102 (2008).

[5] R. Charash, P.P. Maaskant, L. Lewis, C. McAleese, M.J. Kappers, C.J. Humphreys, B. Corbett, Appl. Phys. Lett. 95, 151103 (2009). 